\title{
BMJ Open Efficacy and safety of oral hydroxyurea in transfusion-dependent $\beta$-thalassaemia: a protocol for randomised double-blind controlled clinical trial
}

\author{
Nirmani Yasara, ${ }^{1}$ Nethmi Wickramarathne, ${ }^{1}$ Chamila Mettananda, ${ }^{2}$ \\ Aresha Manamperi, ${ }^{3}$ Anuja Premawardhena, ${ }^{4,5}$ Sachith Mettananda (1) 1,5
}

To cite: Yasara N,

WickramarathneN,MettanandaC et al. Efficacy and safety of oral hydroxyurea in transfusiondependent $\beta$-thalassaemia: a protocol for randomised double-blind controlled clinical trial. BMJ Open 2020;10:e041958. doi:10.1136/ bmjopen-2020-041958

- Prepublication history for this paper is available online. To view these files, please visit the journal online (http://dx.doi. org/10.1136/bmjopen-2020041958).

Received 21 June 2020 Revised 03 October 2020 Accepted 06 0ctober 2020

Check for updates

(c) Author(s) (or their employer(s)) 2020. Re-use permitted under CC BY-NC. No commercial re-use. See rights and permissions. Published by BMJ.

${ }^{1}$ Department of Paediatrics, University of Kelaniya, Ragama, Sri Lanka

${ }^{2}$ Department of Pharmacology, University of Kelaniya, Ragama, Sri Lanka

${ }^{3}$ Molecular Medicine Unit, University of Kelaniya, Ragama, Sri Lanka

${ }^{4}$ Department of Medicine, University of Kelaniya, Ragama, Sri Lanka

${ }^{5}$ Colombo North Teaching Hospital, Ragama, Sri Lanka

Correspondence to

Sachith Mettananda;

sachith.mettananda@kln.ac.lk

\section{ABSTRACT}

Introduction Despite being one of the first diseases to be genetically characterised, $\beta$-thalassaemia remains a disorder without a cure in a majority of patients. Most patients with $\beta$-thalassaemia receive only supportive treatment and therefore have a poor quality of life and shorter life spans. Hydroxyurea, which has shown to induce fetal haemoglobin synthesis in human erythroid cells, is currently recommended for the treatment of sickle cell disease. However, its clinical usefulness in transfusiondependent $\beta$-thalassaemia is unclear. Here, we present a protocol for a randomised double-blind controlled clinical trial to evaluate the efficacy and safety of oral hydroxyurea in transfusion-dependent $\beta$-thalassaemia.

Methods and analysis This single-centre randomised double-blind placebo-controlled clinical trial is conducted at the Thalassaemia Centre of Colombo North Teaching Hospital, Ragama, Sri Lanka. Adult and adolescent patients with haematologically and genetically confirmed transfusion-dependent $\beta$-thalassaemia are enrolled and randomised into the intervention or control group. The intervention group receives oral hydroxyurea $10-20 \mathrm{mg} /$ $\mathrm{kg}$ daily for 6 months, while the control group receives a placebo which is identical in size, shape and colour to hydroxyurea without its active ingredient. Transfused blood volume, pretransfusion haemoglobin level, fetal haemoglobin percentage and adverse effects of treatment are monitored during treatment and 6 months posttreatment. Cessation or reduction of blood transfusions during the treatment period will be the primary outcome measure. The statistical analysis will be based on intention to treat.

Ethics and dissemination Ethical approval has been obtained from the Ethics Committee of Faculty of Medicine, University of Kelaniya (P/116/05/2018) and the trial is approved by the National Medicinal Regulatory Authority of Sri Lanka. Results of the trial will be disseminated in scientific publications in reputed journals.

Trial registration number SLCTR/2018/024; Pre-results.

\section{INTRODUCTION}

$\beta$-Thalassaemia is one of the most common genetic diseases in the world. ${ }^{1}$ It is estimated that 70000 children are born annually with $\beta$-thalassaemia worldwide. ${ }^{2}$ The most severely
Strengths and limitations of this study

- This study evaluates the efficacy of oral hydroxyurea among patients with $\beta$-thalassaemia using a randomised double-blind placebo-controlled clinical trial study design.

- We will evaluate the genetic and clinical characteristics of study participants to identify predictors of response to hydroxyurea treatment.

- This study evaluates the effects of hydroxyurea on multiple outcomes.

- As standard transfusion protocols are continued, this study will not determine the ability of hydroxyurea to maintain a safe but lower steady-state haemoglobin level.

- As we use pretransfusion haemoglobin cut-off of $9.0 \mathrm{~g} / \mathrm{dL}$, this study will not precisely measure changes in fetal haemoglobin percentage.

affected patients develop profound anaemia during infancy, that is life-threatening without blood transfusions. ${ }^{3}$ The only existing cure is allogeneic haematopoietic stem cell transplantation, which is available only to a small subset of patients with human leucocyte antigen matched sibling donors. ${ }^{4}$ All other patients, an overwhelming majority, are managed conservatively with supportive treatment with regular blood transfusions and iron chelation for life. ${ }^{5}$ Due to complications of the disease, these patients experience a poor quality of life and die prematurely in their fourth or fifth decade. ${ }^{78}$

The pathophysiology of $\beta$-thalassaemia centres around the unbalanced synthesis of $\alpha$-like and $\beta$-like globin chains in erythroid cells. ${ }^{9}$ In healthy humans, $\alpha$-globin pairs with $\beta$-globin and $\gamma$-globin to form haemoglobin $(\mathrm{Hb})$ A during postnatal life and $\mathrm{HbF}$ during fetal life, respectively. In $\beta$-thalassaemia, the synthesis of normal $\beta$-globin is markedly reduced due to autosomal recessively inherited mutations of the $\beta$-globin gene. ${ }^{10}$ The resultant 
unbalanced synthesis and accumulation of $\alpha$-globin chains lead to ineffective erythropoiesis and haemolysis, thus cause severe anaemia. ${ }^{11}$ Several genetic modifiers, which include augmentation of the synthesis of $\mathrm{HbF}$ by natural mutations that reactivate $\gamma$-globin, are known to decrease $\alpha$-globin excess and ameliorate the severity of $\beta$-thalassaemia. ${ }^{12}$

Hydroxyurea is a cytostatic agent that interrupts DNA synthesis by inhibiting the ribonucleotide reductase pathway. ${ }^{13}$ It is a well-tolerated Food and Drug Administration (FDA) approved oral medication that is widely used in the treatment of cancers. However, it has been reported as a potent inducer of $\gamma$-globin in human erythroid cells in several preclinical studies. ${ }^{14}{ }^{15}$ It has also shown to be effective as an $\mathrm{HbF}$ inducing agent in patients with sickle cell disease and non-transfusion-dependent $\beta$-thalassaemia. ${ }^{16-18}$ However, the benefit of hydroxyurea in patients with transfusiondependent $\beta$-thalassaemia is equivocal and has not been properly studied in randomised clinical trials. ${ }^{19}$ Two recent Cochrane reviews that analysed the effects of hydroxyurea concluded that the available evidence from clinical trials is insufficient to show hydroxyurea is effective in patients with transfusion-dependent thalassaemia. ${ }^{1720}$ These reviews recommended conducting well-designed randomised controlled clinical trials to evaluate the effects of hydroxyurea in patients with transfusion-dependent $\beta$-thalassaemia. In this paper, we present the protocol for a randomised double-blind placebo-controlled clinical trial to evaluate the efficacy and safety of oral hydroxyurea in transfusiondependent $\beta$-thalassaemia.

\section{METHODS AND ANALYSIS}

\section{Study design and setting}

This study is an ongoing single-centre, randomised, double-blind, placebo-controlled clinical trial to evaluate the efficacy and safety of oral hydroxyurea for transfusiondependent $\beta$-thalassaemia. The study is conducted at the Adult and Adolescent Thalassaemia Centre of Colombo North Teaching Hospital, Ragama, Sri Lanka.

\section{Study hypothesis}

Oral hydroxyurea is an effective, tolerable and safe treatment that upregulates $\mathrm{HbF}$ production, improves pretransfusion haemoglobin level and decreases transfusion requirement in patients with transfusion-dependent $\beta$-thalassaemia.

\section{Study population and eligibility criteria}

Patients with haematologically and genetically confirmed transfusion-dependent $\beta$-thalassaemia attending Adult and Adolescent Thalassaemia Centre of Colombo North Teaching Hospital, Ragama, Sri Lanka will be the study population of this clinical trial.

\section{Inclusion criteria}

- Patients with confirmed genotypes of homozygous/ compound heterozygous $\beta$-thalassaemia major or HbE $\beta$-thalassaemia.
- Patients who are aged over 12 years.

- Patients who required more than eight blood transfusions during the preceding 12 months.

\section{Exclusion criteria}

- Sickle $\beta$-thalassaemia.

- Coexisting chronic liver disease.

- Coexisting chronic kidney disease.

- Coexisting viral hepatitis.

- Patients with contraindications for hydroxyurea (eg, hypersensitivity, bone marrow depression, pregnancy and lactation).

- Patients who are expecting to get pregnant during the next 12 months.

- Patients who have undergone bone marrow transplantation.

- Patients on immunosuppressant therapy.

- Baseline serum ferritin $>5000 \mathrm{ng} / \mathrm{mL}$.

- Baseline white cell count $<4000 / \mu \mathrm{L}$.

- Baseline platelet count $<150000 / \mu \mathrm{L}$.

- Patients who were started on regular transfusions for a predetermined limited period.

\section{Sample size}

The sample size was calculated based on an anticipated response rate of $26 \%$ in the hydroxyurea treatment group reported in previous observational studies, a type I error of 0.05 and a power of $80 \% .{ }^{19}$ The calculated minimum sample size is 50 with 25 patients each in the intervention and control groups. Considering a $10 \%$ dropout rate, we aim to recruit 56 patients for the study.

\section{Subject enrolment, randomisation and blinding}

All eligible patients who fulfil inclusion criteria will be given a patient information sheet to read and time to clarify doubts with investigators before consenting. Informed written consent from all participants will be obtained before recruiting into the study (figure 1). When the patient is below the age of 18 years, consent will be obtained from one of the parents, and assent will be obtained from the patient. At the time of enrolment, information on sociodemographic background, family history, medical history and present medical problems will be gathered using an interviewer-administered questionnaire. Height and weight will be recorded, and abdominal examination will be done to assess hepatic and splenic sizes.

Then patients will be randomised into intervention or placebo group using a stratified block randomisation method. The intervention group will receive hydroxyurea, while the control group will receive a placebo. Each patient will be given a trial number for identification. Hydroxyurea or placebo capsules are packed in sealed envelopes which are labelled with the trial number of each patient at a third-party location, and handed over to the investigators. Participants, data collectors, outcome adjudicators and data analysts will be blinded regarding the treatment until the final analysis of data is available. 


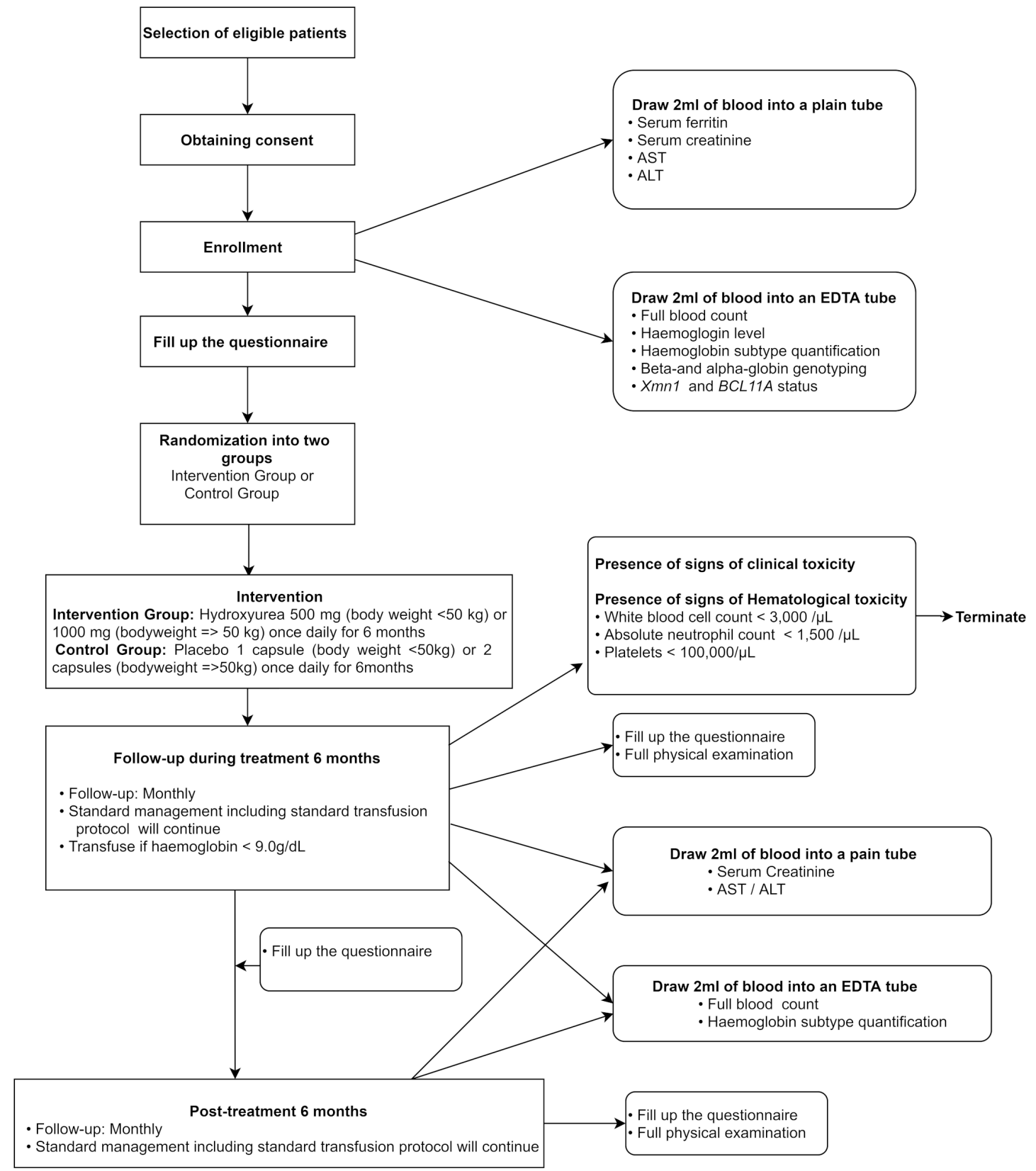

Figure 1 Study design and participant flow through the study. ALT, alanine aminotransferase; AST, aspartate aminotransferase; EDTA, Ethylenediaminetetraacetic acid

\section{Intervention}

Patients in the intervention group will receive oral hydroxyurea (manufactured by Cadila Healthcare Ltd, India) $10-20 \mathrm{mg} / \mathrm{kg}$ daily for 6 months. Patients who weigh less than $50 \mathrm{~kg}$ receive one $500 \mathrm{mg}$ capsule of hydroxyurea, while those who weigh over $50 \mathrm{~kg}$ will receive two $500 \mathrm{mg}$ capsules. Patients in the control group will receive the same number of capsules per body weight of a placebo which is identical in size, shape and colour to hydroxyurea. Placebo capsules (manufactured by State Pharmaceutical Manufacturing Cooperation, Sri Lanka) contain ingredients identical to hydroxyurea except for its active ingredient. All other standard treatments that include blood transfusions and iron chelation will be continued. Patients will receive leuco-depleted packed red blood cell transfusions when haemoglobin drops below $9 \mathrm{~g} / \mathrm{dL}$ as per unit protocol which is in line with the International Thalassaemia Federation Guidelines. ${ }^{4}$

\section{Study procedure}

Clinical evaluation

All patients will be reviewed at least monthly during the 6-month intervention period and followed-up for a further 6 months after discontinuation of treatment. During each review visit, a trained doctor will complete an intervieweradministered questionnaire to assess symptoms of anaemia, known and unknown adverse effects of hydroxyurea and tolerability of hydroxyurea. A complete physical examination will be done to assess weight, height, adverse effects of hydroxyurea and hepatic and splenic sizes. The 
exact volume of transfused blood during each visit will be recorded.

\section{Laboratory evaluation}

Full blood count, haemoglobin subtype quantification, $\alpha$-globin and $\beta$-globin genotyping and Xmn 1 and BCL11A polymorphism status will be performed at enrolment to determine the molecular characteristics of the study population. Additionally, baseline serum ferritin, creatinine, aspartate aminotransferase (AST) and alanine aminotransferase (ALT) levels will be measured at enrolment. During each follow-up visit, full blood count and quantification of $\mathrm{HbF}$ will be performed. Serum ferritin, creatinine, AST and ALT will subsequently be checked at 3-monthly intervals. Soluble transferrin receptor levels will be assessed at the time of enrolment and at the completion of the intervention period.

Full blood counts will be performed using Coulter Ac $\bullet T$ 5 diff CP haematology analyser whereas, quantification of haemoglobin subtypes and variants will be done by capillary electrophoresis using Capillarys 2 Flex Piercing (Sebia) instrument. Serum ferritin, creatinine, AST and ALT will be measured in a clinically accredited laboratory using standard protocols. Soluble transferrin receptor levels will be measured by ELIZA using monoclonal antibodies and a commercially available ELIZA kit (R\&D technologies). DNA will be extracted from the cell pellet using QIAGEN DNA mini kit for $\alpha$-globin and $\beta$-globin genotype assays. Amplification-refractory mutation system PCR method will be used to detect known $\beta$-thalassaemia mutations by manipulating the primer sequence at the $3^{\prime}$ end. Gap-PCR method will be used to detect two common deletional mutations $\left(\alpha_{-}^{-3.7}\right.$ and $\left.\alpha_{-}^{-4.2}\right)$ of the $\alpha$-globin gene and the presence of excess $\alpha$-globin genes using previously published protocols. ${ }^{21} \mathrm{Xmn} 1$ and $B C L 11 \mathrm{~A}$ polymorphisms will be identified by restriction fragment length polymorphism method as per published protocols. ${ }^{22} 23$

\section{Compliance evaluation}

At each review visit, the number of capsules actually taken by patients will be recorded during the 6 -month intervention period. Treatment compliance will be assessed by dividing the actual number of tablets taken by the estimated number of tablets to be taken.

\section{Safety evaluation}

At follow-up visits during the intervention and 6-month postintervention periods, patients will be interviewed by a trained doctor to monitor for known and unknown adverse effects of hydroxyurea treatment. Known adverse effects of hydroxyurea include eczema, skin depigmentation, infection, fever, headache, nausea, vomiting, constipation, gastric discomfort, leg ulcers, mucositis, weight gain, asthma, neutropenia and thrombocytopenia. Both haematological and clinical toxicity will be monitored so that patients could be discontinued from the study if toxicity develops. All study participants will be given contact details of investigators if they wish to clarify any doubts regarding the trial, and to report suspected adverse effects at any point of the trial.

\section{Outcome measures}

Primary outcome

Cessation or reduction of blood transfusion requirement during the treatment period will be the primary outcome measure.

\section{Secondary outcomes}

1. A rise in fetal haemoglobin percentage.

2. Reduction in ineffective erythropoiesis as measured by elevated soluble transferrin receptor levels.

3. Compliance to treatment.

4. Safety and adverse effects of treatment.

\section{Exploratory outcomes}

1. Effect of Xmn1 polymorphism on response to treatment.

2. Effect of BCL11A polymorphism on response to treatment.

\section{Statistical analysis}

Data will be analysed using univariate and multivariate analysis by IBM SPSS statistics V.25. The analysis will be based on intention to treat, and dropouts and discontinued patients will be included in the analysis.

\section{Data management and monitoring}

All completed anonymised questionnaires and laboratory reports will be stored in locked cupboards with participant serial numbers written on individual files. Only the investigators will have access to hard copies. The electronic database will be maintained as a password-protected file. Material obtained from blood samples will be safely destroyed after completion of the study. A three-member independent data monitoring committee comprised of a paediatrician, a haematologist, and a biostatistician has been appointed to make appropriate recommendations on participant safety and trial integrity.

\section{Ethical considerations}

Investigators will not be involved in making management decisions (other than hydroxyurea treatment) which will be done by the clinical team caring for subjects. All decisions regarding blood transfusions will be taken by the clinical management team according to unit protocols. Patients who develop severe adverse events (haematological or clinical) related or unrelated to the treatment during the study period and those who are unable to tolerate hydroxyurea will be discontinued from the study. Suspected adverse events will be reported according to the national guidelines. Participants will have the right to withdraw from the trail at any point without providing explanations.

Ethical approval for the study has been obtained from the Ethics Committee of the Faculty of Medicine, University of Kelaniya, Sri Lanka (Ref. P/116/05/2018). The 
trial is approved by the National Medicinal Regulatory Authority of Sri Lanka.

\section{Termination of the trial}

The trial will be terminated if:

- New information regarding the safety or efficacy of hydroxyurea that indicates a change in the known risk/benefit profile becomes available, such that the risk/benefit is no longer acceptable for subjects participating in the trial.

- Significant violation of good clinical practise that compromises the ability to achieve study objectives or compromises subject safety.

\section{Study status}

The trial commenced in August 2019 according to the protocol version 2.0, 28 May 2018 and is currently open for recruitment. We have recruited 18 patients for the trial so far.

\section{Patient and public involvement}

The research question related to this clinical trial was based on the positive feedback from patients with nontransfusion-dependent $\beta$-thalassaemia who are already taking oral hydroxyurea. Reports of the investigations done as part of the trial will be available to all participants and used in the standard management when required. The results of the study will be disseminated to study participants and all patients with $\beta$-thalassaemia in Sri Lanka using patient education lectures after completion of the trial.

\section{DISCUSSION}

Despite being one of the first genetic diseases to be characterised precisely at the molecular level, $\beta$-thalassaemia remains a life-limiting disorder without an effective cure. ${ }^{24}$ Nonetheless, several attempts exploring novel pathways are currently underway to develop a cure for $\beta$-thalassaemia. Most of these emerging therapies use advanced experimental technologies like gene therapy or genome editing, ${ }^{25-28}$ hence may not be available to a majority of patients living in low-income and middleincome countries. ${ }^{29}$

In this clinical trial, we aim to determine the efficacy and safety of oral hydroxyurea in minimising transfusion requirements and improving clinical outcomes of patients with transfusion-dependent $\beta$-thalassaemia. Hydroxyurea is an already FDA approved drug which is currently being used for other indications including sickle cell disease and non-transfusion-dependent $\beta$-thalassaemia. ${ }^{30}$ However, its efficacy in patients with transfusion-dependent $\beta$-thalassaemia is inconclusive. Our trial is designed to address this knowledge gap regarding treatment of $\beta$-thalassaemia. All previous studies which aim to evaluate the efficacy of hydroxyurea for transfusion-dependent $\beta$-thalassaemia are either observational studies or trials without control arms. ${ }^{19}{ }^{31-34}$ Our study is probably the first randomised double-blind placebo-controlled clinical trial to evaluate the efficacy of hydroxyurea in transfusion-dependent $\beta$-thalassaemia.

Previous use of hydroxyurea has suggested a starting dose of $10-15 \mathrm{mg} / \mathrm{kg} /$ day and dose increments by $5 \mathrm{mg}$ / $\mathrm{kg} /$ day until a maximum tolerable dose of $35 \mathrm{mg} / \mathrm{kg} /$ day is reached. ${ }^{35}{ }^{36}$ Because hydroxyurea is available as $500 \mathrm{mg}$ capsules, we utilised a fixed-dose range of $10-20 \mathrm{mg} / \mathrm{kg}$ / day for this study. After oral administration, hydroxyurea is rapidly absorbed by the gastrointestinal tract, and a peak plasma concentration is detected after 1-4hours. Disproportionately, high peak plasma concentration is associated with increasing dose; hence, once-daily dosing of hydroxyurea is recommended. ${ }^{36}$

The effects of hydroxyurea in $\beta$-thalassaemia could be multiple. Therefore, in the current study, we aim to evaluate the effects of hydroxyurea on transfusion requirement, pretransfusion haemoglobin, $\mathrm{HbF}$ levels and ineffective erythropoiesis. Additionally, we will evaluate several factors, for example $\alpha$-globin and $\beta$-globin genotypes and genetic polymorphisms in the Xmn1 and $B C L 11 A$ genomic loci, which may be important in determining the response to hydroxyurea. This is particularly relevant as polymorphisms in BCL11A and Xmn1 have been suggested to predict the response to hydroxyurea in non-transfusion-dependent $\beta$-thalassaemia patients. ${ }^{37} 38$ Therefore, through these approaches, we will be able to provide important mechanistic data on the action of hydroxyurea which is still incomplete.

\section{LIMITATIONS}

One important limitation of this trial is that these patients are on regular transfusion regimens. It was deemed unethical to stop transfusions in these patients for us to evaluate the full efficacy of hydroxyurea to see whether these patients could maintain a steady-state, safe although lower haemoglobin level of approximately $7-8 \mathrm{~g} / \mathrm{dL}$ without transfusions. All patients in the trial will continue to receive standard treatment with transfusions when their haemoglobin drops below $9 \mathrm{~g} / \mathrm{dL}$. Therefore, we will only be able to evaluate the reduction in transfusion requirement as an outcome measure. Similarly, we may not be able to evaluate the effects on $\mathrm{HbF}$ fully. However, this is acceptable as most previous clinical trials on transfusiondependent $\beta$-thalassaemia have demonstrated reduction in transfusion burden rather than complete cessation of transfusions. ${ }^{39}$

Contributors AP and SM conceived the study. NY, NW, CM, AM, AP and SM contributed to the study design. NY drafted the manuscript and CM and SM finalised the manuscript. All authors assisted in developing the protocol and have read, reviewed, edited and approved the final manuscript.

Funding This work was supported by the National Research Council of Sri Lanka Investigator Driven Research Grant (Number 18-030) to SM. Funding agency does not have any role in the study design, data collection, data analysis or publication of results.

Competing interests None declared.

Patient consent for publication Not required. 
Provenance and peer review Not commissioned; externally peer reviewed.

Open access This is an open access article distributed in accordance with the Creative Commons Attribution Non Commercial (CC BY-NC 4.0) license, which permits others to distribute, remix, adapt, build upon this work non-commercially, and license their derivative works on different terms, provided the original work is properly cited, appropriate credit is given, any changes made indicated, and the use is non-commercial. See: http://creativecommons.org/licenses/by-nc/4.0/.

ORCID iD

Sachith Mettananda http://orcid.org/0000-0002-0760-0418

\section{REFERENCES}

1 Taher AT, Weatherall DJ, Cappellini MD. Thalassaemia. Lancet 2018;391:155-67.

2 Weatherall DJ. The inherited diseases of hemoglobin are an emerging global health burden. Blood 2010;115:4331-6.

3 Mettananda S. Management of thalassaemia. Sri Lanka J Child Health 2018;47:159-65.

4 Cappellini MD, Cohen A, Porter J, et al. Guidelines for the management of transfusion dependent thalassaemia (TdT). 3rd edn. Cyprus: Thalassaemia International Federation, 2014.

5 Mettananda S, Pathiraja H, Peiris R, et al. Blood transfusion therapy for $\beta$-thalassemia major and hemoglobin $E \beta$-thalassemia: adequacy, trends, and determinants in Sri Lanka. Pediatr Blood Cancer 2019;66:e27643.

6 Suriapperuma T, Peiris R, Mettananda C, et al. Body iron status of children and adolescents with transfusion dependent $\beta$-thalassaemia: trends of serum ferritin and associations of optimal body iron control. BMC Res Notes 2018;11:547.

7 Mettananda S, Peiris R, Pathiraja H, et al. Psychological morbidity among children with transfusion dependent $\beta$-thalassaemia and their parents in Sri Lanka. PLoS One 2020;15:e0228733.

8 Mettananda S, Pathiraja $H$, Peiris $R$, et al. Health related quality of life among children with transfusion dependent $\beta$-thalassaemia major and haemoglobin $\mathrm{E} \beta$-thalassaemia in Sri Lanka: a case control study. Health Qual Life Outcomes 2019;17:137.

9 Mettananda S, Gibbons RJ, Higgs DR. Understanding $\alpha$-globin gene regulation and implications for the treatment of $\beta$-thalassemia. Ann $N$ Y Acad Sci 2016;1368:16-24.

10 Mettananda S, Higgs DR. Molecular basis and genetic modifiers of thalassemia. Hematol Oncol Clin North Am 2018;32:177-91.

11 Mettananda S, Gibbons RJ, Higgs DR. $\alpha$-Globin as a molecular target in the treatment of $\beta$-thalassemia. Blood 2015;125:3694-701.

12 Bauer DE, Kamran SC, Orkin SH. Reawakening fetal hemoglobin: prospects for new therapies for the $\beta$-globin disorders. Blood 2012;120:2945-53.

13 Kosaryan M, Karami H, Zafari M, et al. Report on patients with non transfusion-dependent $\beta$-thalassemia major being treated with hydroxyurea attending the thalassemia research center, Sari, Mazandaran Province, Islamic Republic of Iran in 2013. Hemoglobin 2014;38:115-8

14 Fucharoen S, Siritanaratkul N, Winichagoon P, et al. Hydroxyurea increases hemoglobin $\mathrm{F}$ levels and improves the effectiveness of erythropoiesis in beta-thalassemia/hemoglobin $\mathrm{E}$ disease. Blood 1996;87:887-92.

15 Letvin NL, Linch DC, Beardsley GP, et al. Augmentation of fetalhemoglobin production in anemic monkeys by hydroxyurea. $N$ Engl J Med 1984;310:869-73.

16 Algiraigri $\mathrm{AH}$, Kassam A. Hydroxyurea for hemoglobin $\mathrm{E} / \mathrm{\beta}-$ thalassemia: a systematic review and meta-analysis. Int $\mathrm{J}$ Hematol 2017:106:748-56.

17 Foong WC, Ho JJ, Loh CK, et al. Hydroxyurea for reducing blood transfusion in non-transfusion dependent beta thalassaemias. Cochrane Database Syst Rev 2016;10:CD011579.
18 Nevitt SJ, Jones AP, Howard J, et al. Hydroxyurea (hydroxycarbamide) for sickle cell disease. Cochrane Database Syst Rev 2017;4:CD002202.

19 Algiraigri $\mathrm{AH}$, Wright NAM, Paolucci EO, et al. Hydroxyurea for lifelong transfusion-dependent $\beta$-thalassemia: a meta-analysis. Pediatr Hematol Oncol 2017;34:435-48.

20 Ansari SH, Lassi ZS, Khowaja SM, et al. Hydroxyurea (hydroxycarbamide) for transfusion-dependent $\beta$-thalassaemia. Cochrane Database Syst Rev 2019;3:CD012064.

21 Premawardhena A, Allen A, Piel F, et al. The evolutionary and clinical implications of the uneven distribution of the frequency of the inherited haemoglobin variants over short geographical distances. $\mathrm{Br}$ J Haematol 2017;176:475-84.

22 Perera S, Allen A, Silva I, et al. Genotype-Phenotype association analysis identifies the role of $\alpha$ globin genes in modulating disease severity of $\beta$ thalassaemia intermedia in Sri Lanka. Sci Rep 2019;9:10116.

23 Peri KG, Gagnon J, Gagnon C, et al. Association of $-158(\mathrm{C} \rightarrow \mathrm{T})$ (Xmnl) DNA polymorphism in G gamma-globin promoter with delayed switchover from fetal to adult hemoglobin synthesis. Pediatr Res 1997;41:214-7.

24 Cappellini MD, Porter JB, Viprakasit V, et al. A paradigm shift on beta-thalassaemia treatment: how will we manage this old disease with new therapies? Blood Rev 2018;32:300-11.

25 Thompson AA, Walters MC, Kwiatkowski J, et al. Gene therapy in patients with transfusion-dependent $\beta$-thalassemia. $N$ Engl $\mathrm{J}$ Med 2018;378:1479-93.

26 Mettananda S, Yasara N, Fisher CA, et al. Synergistic silencing of $\alpha$-globin and induction of $\gamma$-globin by histone deacetylase inhibitor, vorinostat as a potential therapy for $\beta$-thalassaemia. Sci Rep 2019:9:11649.

27 Mettananda S, Fisher CA, Hay D, et al. Editing an $\alpha$-globin enhancer in primary human hematopoietic stem cells as a treatment for $\beta$-thalassemia. Nat Commun 2017:8:424.

28 Mettananda S, Fisher CA, Sloane-Stanley JA, et al. Selective silencing of $\alpha$-globin by the histone demethylase inhibitor IOX1: a potentially new pathway for treatment of $\beta$-thalassemia. Haematologica 2017;102:e80-4.

29 Mettananda S. Thalassaemia: in a quest towards an ultimate cure. Sri Lanka J Child Health 2017;46:203-10.

30 Algiraigri AH, Wright NAM, Paolucci EO, et al. Hydroxyurea for nontransfusion-dependent $\beta$-thalassemia: a systematic review and meta-analysis. Hematol Oncol Stem Cell Ther 2017;10:116-25.

31 Bradai M, Abad MT, Pissard S, et al. Hydroxyurea can eliminate transfusion requirements in children with severe beta-thalassemia. Blood 2003;102:1529-30.

32 Ansari SH, Shamsi TS, Munzir S, et al. G $\gamma-X m n$ I polymorphism: a significant determinant of $\beta$-thalassemia treatment without blood transfusion. J Pediatr Hematol Oncol 2013;35:e153-6.

33 Ansari SH, Shamsi TS, Ashraf M, et al. Efficacy of hydroxyurea in providing transfusion independence in $\beta$-thalassemia. $J$ Pediatr Hematol Oncol 2011;33:339-43.

34 Karimi M, Haghpanah S, Farhadi A, et al. Genotype-phenotype relationship of patients with $\beta$-thalassemia taking hydroxyurea: a 13year experience in Iran. Int J Hematol 2012;95:51-6.

35 Estepp JH, Smeltzer MP, Kang G, et al. A clinically meaningful fetal hemoglobin threshold for children with sickle cell anemia during hydroxyurea therapy. Am J Hematol 2017;92:1333-9.

36 Agrawal RK, Patel RK, Shah V, et al. Hydroxyurea in sickle cell disease: drug review. Indian J Hematol Blood Transfus 2014;30:91-6.

37 Banan M. Hydroxyurea treatment in $\beta$-thalassemia patients: to respond or not to respond? Ann Hematol 2013;92:289-99.

38 Pule GD, Mowla S, Novitzky N, et al. Hydroxyurea down-regulates BCL11A, KLF-1 and Myb through miRNA-mediated actions to induce $\gamma$-globin expression: implications for new therapeutic approaches of sickle cell disease. Clin Transl Med 2016;5:15.

39 Cappellini MD, Viprakasit V, Taher AT, et al. A phase 3 trial of Luspatercept in patients with transfusion-dependent $\beta$-thalassemia. N Engl J Med 2020;382:1219-31. 